\title{
ERRATUM
}

\section{Scalable 2-Pass Data Mining Technique for Large Scale Spatio-temporal Datasets}

\author{
Tahar Kechadi and Michela Bertolotto \\ University College Dublin, Belfield, Dublin 4, Ireland. \\ \{tahar.kechadi, michela.bertolotto\} @ucd.ie \\ Sergio Di Martino, Filomena Ferrucci \\ Dip. di Matematica e Informatica, Università degli Studi di Salerno, Italy \\ \{sdimartino, fferrucci\}@unisa.it \\ B. Apolloni et al. (Eds.): KES 2007/ WIRN 2007, Part II, LNAI 4693, pp. 785-792, 2007.
}

(C) Springer-Verlag Berlin Heidelberg 2007

\section{DOI 10.1007/978-3-540-74827-4_171}

The names of the third and fourth authors have been incorrectly positioned between the affiliations. Consequently, these names are missing from the table of contents, author index, running heads, and metadata. The correct positioning is given below:

Tahar Kechadi ${ }^{1}$, Michela Bertolotto ${ }^{1}$, Sergio Di Martino ${ }^{2}$, Filomena Ferrucci ${ }^{2}$

\footnotetext{
${ }^{1}$ University College Dublin, Belfield, Dublin 4, Ireland \{tahar.kechadi, michela.bertolotto\} @ucd.ie

${ }^{2}$ Dip. di Matematica e Informatica, Università degli Studi di Salerno, Italy \{sdimartino, fferrucci\} @unisa.it
} 